\title{
The Acute Effect of Adequate Water Intake on Glucose Regulation in Low Drinkers
}

\author{
Adam Seal $^{\mathrm{a}}$ Abigail T. Colburn ${ }^{\mathrm{b}}$ HyunGyu Suh ${ }^{\mathrm{c}}$ Stavros A. Kavouras ${ }^{\mathrm{b}}$

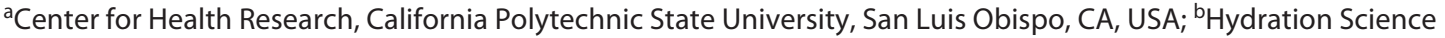 \\ Laboratory, Arizona State University, Phoenix, AZ, USA; 'Exercise Physiology Laboratory, Georgia Institute of \\ Technology, Atlanta, GA, USA
}

\section{Keywords}

Glucose regulation · Hydration · Vasopressin

\begin{abstract}
Background: Arginine vasopressin (AVP), a key hormone in fluid balance, may be a modifiable contributor to hyperglycemia [1]. Low daily water drinkers often exhibit increased urine concentration and copeptin, a surrogate marker for AVP $[2,3]$. Objective: The primary purpose was to investigate the acute effect of adequate water intake on daily glucose concentration in low drinkers. Secondarily, the study examined if adequate water intake could improve glucoregulatory hormonal profiles in low drinkers. Methods: Seven healthy (5 males, 2 females; age $43 \pm$ 7 years, BMI $31 \pm 3$ ) low drinkers were recruited using a water frequency questionnaire and a 24-h urine sample. Participants were recruited using social media channels and flyers in local community. Classification of a low drinker was defined by a fluid

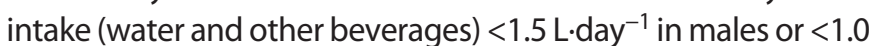
L.day ${ }^{-1}$ in females and a $24-\mathrm{h} \cdot$ UOsm of $>800 \mathrm{mmol} \cdot \mathrm{kg}^{-1}$. In a crossover counterbalanced design, participants remained in the laboratory for $11 \mathrm{~h}$ (07:00-18:00) and were provided either the Institute of Medicine's recommended amount of water excluding fluid from food (males: $3 \mathrm{~L} \cdot$ day $^{-1}$, females: $2 \mathrm{~L} \cdot$ day $^{-1}$; high water intake, $\mathrm{HWI}$ ) or an amount representing the bottom quartile of water consumption observed in the National Health and Nutrition Examination Survey (males: 0.5 L.day $^{-1}$, females: 0.4 L.day ${ }^{-1}$; low iater intake, LWI) (Table 1) [4, 5]. Caloric intake was standardized to body weight $\left(100 \mathrm{~kJ} \cdot \mathrm{kg}^{-1}\right)$ with an identical ratio
\end{abstract}

karger@karger.com www.karger.com/anm

Karger!
(C) 2022 The Author(s)

Published by S. Karger AG, Basel

This article is licensed under the Creative Commons Attribution 4.0 International License (CC BY) (http://www.karger.com/Services/ OpenAccessLicense). Usage, derivative works and distribution are permitted provided that proper credit is given to the author and the original publisher. of macronutrients and time of consumption between trials (Table 1). At 07:00, fasted baseline blood was drawn. Subsequent blood draws performed across the next 11 hours were analyzed for copeptin, glucose, insulin, glucagon, cortisol, and GLP-1 (Table 1). All urine voids during the 11-h protocol were pooled and analyzed for osmolality and glucose $(n=4)$. A two-way (water intake $\times$ time) repeated-measures ANOVA was used to determine differences in hydration and glucoregulatory measures. Dependent $t$ tests were used to measure differences in urine samples. Statistical significance was determined a priori at an alpha of 0.05 . Results: Participants were confirmed as low drinkers according to daily fluid intake, 24-h.UOsm, and copeptin (water frequency questionnaire volume: $823 \pm 403 \mathrm{~mL} \cdot$ day $^{-1}$, 24-h.UOsm: $961 \pm 105 \mathrm{mmol} \cdot \mathrm{kg}^{-1}$, copeptin:8.17 $\pm 3.05 \mathrm{pmol} \cdot \mathrm{L}^{-1}$ ). During the experiments, 11 -h.UOsm (HWl: $224 \pm 48 \mathrm{mmol} \cdot \mathrm{kg}^{-1}$, LWI: $\left.956 \pm 120 \mathrm{mmol} \cdot \mathrm{kg}^{-1}\right)$, plasma osmolality, and copeptin were lower in HWI as than in LWI $(p=<0.05$, Fig. 1). There was a borderline significant main effect of water intake on plasma glucose ( $p=0.07$, Fig. 2 ) and total urinary glucose output (HWl: 51.4 $\pm 6.9 \mathrm{mg}$, LWl: $40.1 \pm 10.4 \mathrm{mg}, p=0.07)$. Cortisol was significantly higher in LWI as than in HWI ( $p=0.009$, Fig. 2); however, no pairwise differences were observed in post hoc analysis. Glucagon, insulin, and GLP-1 were similar between trials $(p>0.05)$. Conclusion: Acute increases in water intake may mildly reduce daily plasma glucose concentrations in low drinkers. This may be due to acutely increased urinary glucose output when low drinkers are given adequate amounts of water. Increased water intake also led to decreased cortisol concentration.

(C) 2022 The Author(s).

Published by S. Karger AG, Basel

Correspondence to:

Adam Seal, adseal@calpoly.edu 
Table 1. Water intake, meals, and blood draws during experimental protocol

\begin{tabular}{|c|c|c|c|c|c|c|}
\hline \multirow[t]{3}{*}{ Time } & \multicolumn{4}{|c|}{ Water intake, $\mathrm{mL}$} & \multirow[t]{3}{*}{ Meal } & \multirow[t]{3}{*}{ Blood draw } \\
\hline & \multicolumn{2}{|l|}{ male } & \multicolumn{2}{|c|}{ female } & & \\
\hline & $\mathrm{HWI}$ & LWI & $\mathrm{HWI}$ & LWI & & \\
\hline 07:00 & 500 & 100 & 250 & 50 & Breakfast & Yes \\
\hline 08:00 & 250 & - & 125 & - & - & Yes \\
\hline 09:00 & 250 & 100 & 125 & 100 & - & Yes \\
\hline 10:00 & 250 & - & 250 & - & Snack 1 & - \\
\hline 11:00 & 250 & - & 125 & - & - & - \\
\hline $12: 00$ & 250 & 50 & 250 & 50 & Lunch & Yes \\
\hline 13:00 & 250 & - & 125 & - & - & Yes \\
\hline $14: 00$ & 250 & 100 & 250 & 100 & Snack 2 & Yes \\
\hline $15: 00$ & 250 & - & 125 & - & - & - \\
\hline $16: 00$ & 250 & 50 & 250 & 50 & Dinner & Yes \\
\hline $17: 00$ & 250 & 100 & 125 & 50 & - & Yes \\
\hline $18: 00$ & - & - & - & - & - & Yes \\
\hline
\end{tabular}

HWI, high water intake; LWI, low water intake.
Fig. 1. Plasma osmolality (a) and copeptin (b) during HWI (males: 3 L; females: 2 L) and LWI (males: $1 \mathrm{~L}$; females: $0.5 \mathrm{~L}$ ) trials. *Represents significantly different from HWI for time point $(\mathrm{p}<0.05)$. Error bars $=$ SE. HWI, high water intake; LWI, low water intake.

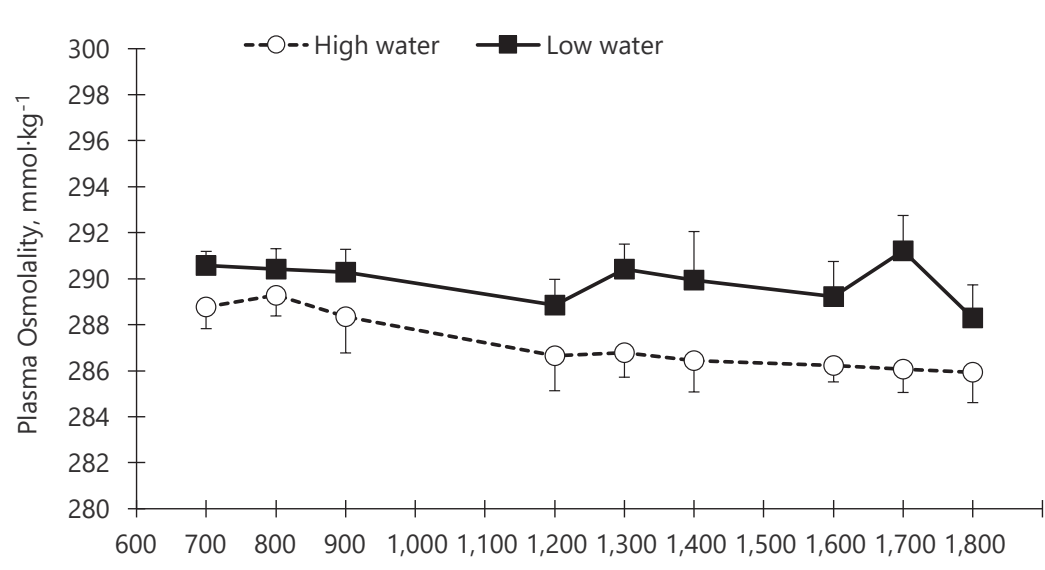

Time, hours

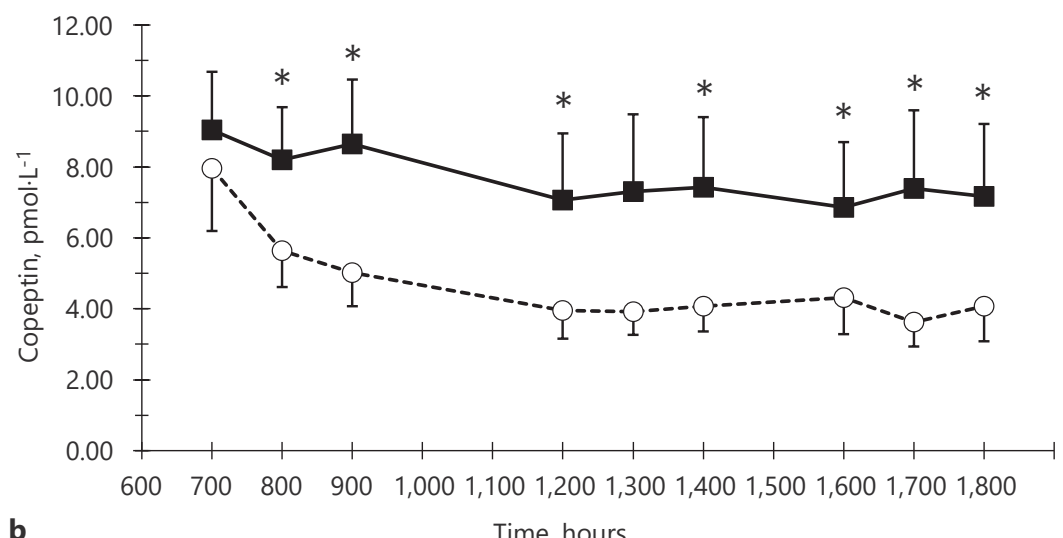

b 
Fig. 2. Significant main effect of water intake on cortisol $(p<0.05)(\mathbf{a})$ and borderline significant main effect of water intake on plasma glucose $(p=0.07)(\mathbf{b})$ during HWI (males: 3 L; females: 2 L) and LWI (males: $1 \mathrm{~L}$; females: $0.5 \mathrm{~L}$ ) trials. Error bars = SE. HWI, high water intake; LWI, low water intake.

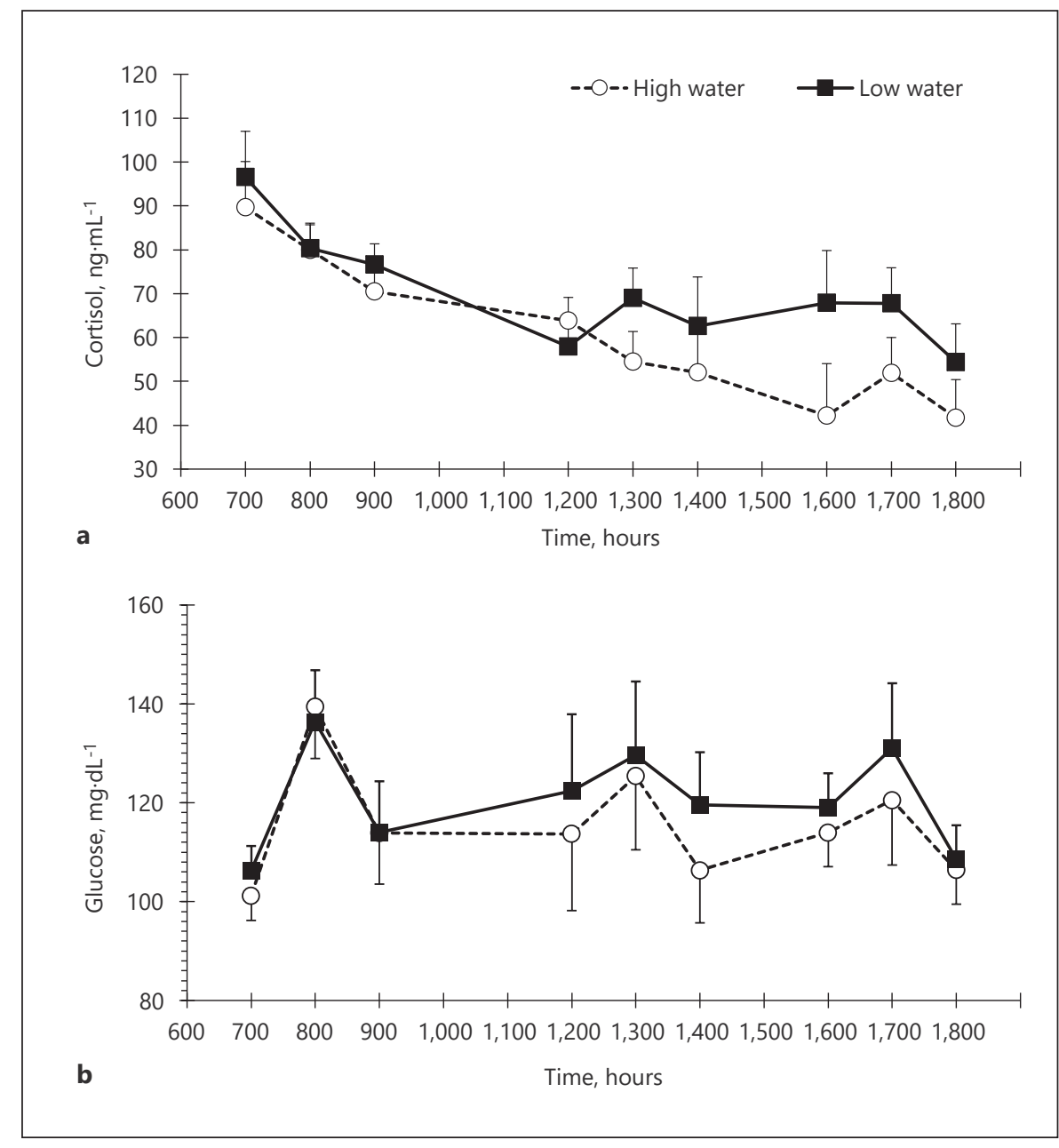

\section{Acknowledgement}

The authors would like to thank Ginger Hook and Veronica Zamora for their dedication and assistance during data collection.

\section{Statement of Ethics}

The research was conducted ethically in accordance with the World Medical Association Declaration of Helsinki. Subjects gave their written informed consent and the study protocol was approved by the Arizona State University Institutional Review Board, approval number STUDY00010276.

\section{Conflict of Interest Statement}

S.A.K. has served as scientific consultant for Quest Diagnostics, Standard Process, and Danone Research and has received grants from Danone Research and Standard Process. A.S. has received speakers fees from Danone Research.

Effect of Adequate Water Intake on Glucose Regulation

\section{Funding Sources}

This research received no funding.

\section{Author Contributions}

All authors designed the study; A.S., A.T.C., and H.S. conducted data collection and sample analysis; A.S. and S.A.K. analyzed the data; A.S. wrote the paper; S.A.K. was the principal investigator. All authors read, critically revised, and approved the final manuscript.

\section{Data Availability Statement}

The data that support the findings of this study are available from the corresponding author, A.S., upon reasonable request. 


\section{References}

1 Balanescu S, Kopp P, Gaskill MB, Morgenthaler NG, Schindler C, Rutishauser J. Correlation of plasma copeptin and vasopressin concentrations in hypo-, iso-, and hyperosmolar States. J Clin Endocrinol Metab. 2011; 96(4):1046-52.

2 Perrier E, Vergne S, Klein A, Poupin M, Rondeau P, Le Bellego L, et al. Hydration biomarkers in free-living adults with different levels of habitual fluid consumption. $\mathrm{Br}$ Nutr. 2013;109(9):1678-87.
3 Lemetais G, Melander O, Vecchio M, Bottin $\mathrm{JH}$, Enhörning S, Perrier ET. Effect of increased water intake on plasma copeptin in healthy adults. Eur J Nutr. 2018. 57(5):188390.

4 Institute of Medicine. Dietary reference intakes for water, potassium, sodium, chloride, and sulfate; 2005. p. 610

5 Drewnowski A, Rehm CD, Constant F. Water and beverage consumption among adults in the United States: cross-sectional study using data from NHANES 2005-2010. BMC Public Health. 2013;13:1068. 